\title{
STAT3 regulates the migration and invasion of a stem-like subpopulation through microRNA-21 and multiple targets in hepatocellular carcinoma
}

\author{
NING ZHANG ${ }^{1,2^{*}}$, WEI-DONG DUAN ${ }^{1 *}$, JIAN-JUN LENG $^{1 *}$, LIANG ZHOU $^{3 *}$, XING WANG $^{2}$, \\ YIN-ZHE XU ${ }^{1}$, XUE-DONG WANG ${ }^{1}$, AI-QUN ZHANG ${ }^{1}$ and JIA-HONG DONG ${ }^{1}$
}

\author{
${ }^{1}$ Institute of Hepatobiliary Surgery and Liver Transplantation Center, China General Hospital of PLA, Beijing 100853; \\ ${ }^{2}$ Department of Hepatobiliary Surgery, Xijing Hospital, The Fourth Military Medical University, Xi'an, Shannxi 710032; \\ ${ }^{3}$ Department of General Surgery, The 155 Central Hospital of PLA, Kaifeng, He'nan 471000, P.R. China
}

Received November 5, 2014; Accepted December 12, 2014

DOI: $10.3892 /$ or.2015.3710

\begin{abstract}
Despite advances in the detection and treatment of hepatocellular carcinoma (HCC), the prognosis remains poor partly due to recurrence or extra/intrahepatic metastasis. Stem-like cancer cells are considered the source of malignant phenotypes including metastasis in various types of cancer. HCC side population (SP), considered as stem-like cancer cells, plays an important role in the migration and invasion in HCC, while the mechanisms involved remain unknown. In the present study, high levels of STAT3 and phospho-STAT3 were observed in MHCC97H SP cells compared with the main population (MP) cells. Inhibition of phospho-STAT3 led to a reduction of miR-21 expression, an increase of PTEN, RECK, and programmed cell death 4 (PDCD4) expression as well as the migration and invasion of SP cells. A set of rescue experiments was performed using different combinations of STAT3 inhibitor, miR-21 mimics and siRNAs to observe the expression of miR-21 targets, cell migration and invasion alterations. Data indicated that the alterations induced by STAT3 inhibition were partly reversed by the upregulation of miR-21. Additionally, the cells migration and invasion when silencing the targets of miR-21 were also reversed by STAT3 inhibition. In conclusion, the present study revealed the aberrant expression of STAT3 and miR-21 in HCC SP cells. Targeting STAT3 may limit HCC migration and invasion, which is likely to involve the regulation of miR-21 and its targets PTEN, RECK
\end{abstract}

Correspondence to: Professor Jia-Hong Dong, Institute of Hepatobiliary Surgery and Liver Transplantation Center, China General Hospital of PLA, No. 28 Fuxing road, Haidian District, Beijing 100853, P.R. China

E-mail: dongjh301@163.com

\section{*Contributed equally}

Key words: hepatocellular carcinoma, side population, STAT3, microRNA-21, metastasis and PDCD4. Strategies directed towards STAT3 may therefore be a novel approach for the treatment of HCC.

\section{Introduction}

Hepatocellular carcinoma (HCC) is the sixth most common cancer, and it is the third leading cause of cancer mortality worldwide (1). Despite advances in the detection and treatment of HCC, the mortality remains high because the majority of HCC patients present at an advanced stage or with metastasis for which most potentially curative therapies have limited efficacy (2). Thus, understanding the mechanism underlying carcinogenesis and metastatic formation and treating invasion and metastasis as therapeutic targets are essential for the management of liver malignancies.

Cancer stem cell (CSC) hypothesis assumes that a small amount of cancer cells with stem-like characteristics, including self-renewal and differentiation to a particular lineage of mature cells (3), can initiate and sustain tumor growth, drive relapse (4) and be responsible for early systemic dissemination and metastasis formation (5). The side population (SP) phenotype is thought to be enriched for stem-like cells in normal tissues and various types of cancer. Previously, the identification and isolation of SP cells in human HCC cell lines was reported and its stem-like characteristics compared with main population (MP) cells, including quiescence, elevated chemoresistance, increased tumorigenicity, higher actin polymerization ability and increased migration capacity towards the chemokine CXCL12 (6) were confirmed.

Signal transducer and activator of transcription 3 (STAT3) is a transcription factor that regulates various genes involved in different biological and tumorigenic processes. In response to cytokines or environmental factors, STAT3 becomes phosphorylated on Tyr-705 and/or Ser-727, resulting in enhanced transcriptional activity, making it a potent inducer of proliferation, anchorage-independent growth, tumorigenesis, invasion, metastasis and angiogenesis (7-9).

As a downstream regulator of the STAT3 signaling pathway, STAT3 phosphorylation is also involved in the regulation of microRNAs (10), which can negatively regulate 
various protein expression levels at the post-transcriptional level by translational inhibition and/or mRNA degradation. It was reported that STAT3 bound to multiple sites in miR-21 promoter and was necessary for miR-21 expression and induction of transcription $(11,12)$. It has been widely demonstrated that miR-21 can function as an oncogene and increase tumor cell migration and invasion by directly targeting PTEN, RECK and programmed cell death 4 (PDCD4) $(13,14)$. A higher miR-21 expression in HCC SP cells compared with MP cells was previously identified (15), as well as the fact that repression of miR-21 inhibited SP cell migration and invasion in vitro, possibly due to the downregulation of the tumor suppressor PTEN, RECK or PDCD4.

Considering STAT3, miR-21 and its targets have been widely explored as cancer-related targets for tumors including HCC, the effects of STAT3 on the metastasis-related capacities of HCC SP cells, and whether the effects were mediated through miR-21 and its targets were determined. To the best of our knowledge, no information is available concerning this issue. Thus, we conducted the present study to determine the effects and potential mechanism of STAT3 in the metastasis of HCC SP cells.

\section{Materials and methods}

Cell culture and cell sorting. The MHCC97H human HCC cell line was cultured in DMEM medium supplemented with $10 \%$ fetal calf serum (Sigma Chemical Co., St. Louis, MO, USA) and $1 \%$ penicillin-streptomycin G (Invitrogen Life Technologies, Carlsbad, CA, USA). In all experiments, the cells were cultured at $37^{\circ} \mathrm{C}$ in a humidified $5 \% \mathrm{CO}_{2} / 95 \%$ air atmosphere. To identify and isolate the SP and MP fractions, we used flow cytometric analysis as described previously (6). Briefly, the cells were preincubated at $37^{\circ} \mathrm{C}$ for $15 \mathrm{~min}$ and stained with Hoechst 33342 dye (Sigma Chemical Co.) at $6 \mu \mathrm{g} / \mathrm{ml}$. The cells were then incubated for $90 \mathrm{~min}$ at $37^{\circ} \mathrm{C}$ alone or with $50 \mu \mathrm{M}$ verapamil (Sigma Chemical Co.), and then stained with $2 \mu \mathrm{g} / \mathrm{ml}$ of propidium iodide (BD Pharmingen, San Diego, CA, USA) to label dead cells. The cells were then filtered through a $40-\mu \mathrm{m}$ strainer (BD Falcon) and maintained at $4^{\circ} \mathrm{C}$ before analysis and sorting using a FACSAria flow cytometer (BD Falcon). Samples of sorted cells were reanalyzed to examine the sorting purities.

Quantitative RT-PCR. Total RNA, including miRNAs, was isolated from MCHH97H cells with TRIzol reagent (Invitrogen) according to the manufacturer's instructions. Expression of hsa-miR-21 was analyzed with the miScript system (Qiagen, Valencia, CA, USA), which consists of the miScript Reverse Transcription kit, miScript Primer assays and miScript SYBR-Green PCR kit, according to the manufacturer's instructions. Small nuclear RNA U6 was used for normalization. Quantitative RT-PCR (RT-qPCR) was run on the ABI PRISM 7700 Sequence Detector (Applied Biosystems, Foster City, CA, USA). Reactions were run in triplicate. The $\Delta \Delta \mathrm{Ct}$ method was used for relative quantification of the gene expression to determine miR-21 expression levels.

Protein extraction and western blotting. The cells were lysed in lysis buffer as previously described (15) by incubating for
$20 \mathrm{~min}$ at $4^{\circ} \mathrm{C}$. The protein concentration was determined using the Bio-Rad assay system (Bio-Rad Laboratories, Hercules, CA, USA). Total proteins were fractionated using SDS-PAGE and transferred onto nitrocellulose membranes. The membranes were blocked with $5 \%$ non-fat dried milk or bovine serum albumin in $1 \mathrm{X}$ TBS buffer containing $0.1 \%$ Tween-20 and then incubated with the appropriate primary antibodies. Horseradish peroxidase-conjugated anti-rabbit or anti-mouse IgG was used as the secondary antibody and the protein bands were detected using the enhanced chemiluminescence detection system (Amersham Pharmacia Biotech, Amersham, UK). Quantification of the western blots was performed using laser densitometry and the relative protein expression was then normalized to glyceraldehyde-3-phosphate dehydrogenase (GAPDH) levels.

Apoptosis assays. JSI-124 (cucurbitacin-I, Santa Cruz Biotechnology, Inc., Santa Cruz, CA, USA) was dissolved in DMSO. Cells were treated with the appropriate volume of DMSO for the vehicle control. Following treatment with different concentrations of JSI-124 or DMSO, the cells were washed twice in PBS and resuspended in $500 \mu \mathrm{l}$ of $1 \mathrm{X}$ binding buffer prior to incubation with $5 \mu \mathrm{l}$ of Annexin $\mathrm{V}$ and $10 \mu \mathrm{l}$ of PI. The cells were then analyzed by using flow cytometry after incubation for 5-10 min in the dark. Early apoptotic cells were stained with Annexin V alone whereas necrotic and late apoptotic cells were stained with both Annexin V and PI.

Actin polymerization. SP cells were resuspended and maintained at $37^{\circ} \mathrm{C}$. Human CXCL12 (Peprotech, Inc., Rocky Hill, NJ, USA) or PBS were added to cell suspensions and aliquots were removed at the indicated times and immediately fixed in 4\% paraformaldehyde for $10 \mathrm{~min}$. After washing, the samples were stained with FITC Phalloidin (Molecular Probes, Eugene, OR, USA) stain F-actin and analyzed by flow cytometry. The relative F-actin index was determined as the ratio of the F-actin level of different cells treated with CXCL12 to cells treated with PBS.

Migration and invasion assays. Cell migration was analyzed with non-Matrigel-coated Transwell cell culture chambers (Millipore, Billerica, MA, USA). Cell invasion was analyzed with Matrigel-coated Transwell cell culture chambers (Millipore). Cells treated with different reagents $\left(5 \times 10^{4}\right.$ cells/well) were serum-starved for $24 \mathrm{~h}$ and plated in the upper insert of a 24-well chamber in serum-free medium. A medium with or without CXCL12 chemokine was added to the well. After incubation for $24 \mathrm{~h}$, the cells on the upper side of the filters were mechanically removed by scrubbing with a cotton swab, after which the membrane was fixed with $4 \%$ formaldehyde for $10 \mathrm{~min}$ at room temperature and stained with $0.5 \%$ crystal violet for $10 \mathrm{~min}$. Invasive or migrated cells were counted at a magnification of x200 from 10 different fields of each filter.

Statistical analysis. Each experiment was repeated at least three times. The data were summarized and presented as means \pm SD. The differences among means were statistically analyzed using a t-test. Statistical analyses were performed using SPSS 13.0 software (Chicago, IL, USA). $\mathrm{P}<0.05$ was considered statistically significant. 


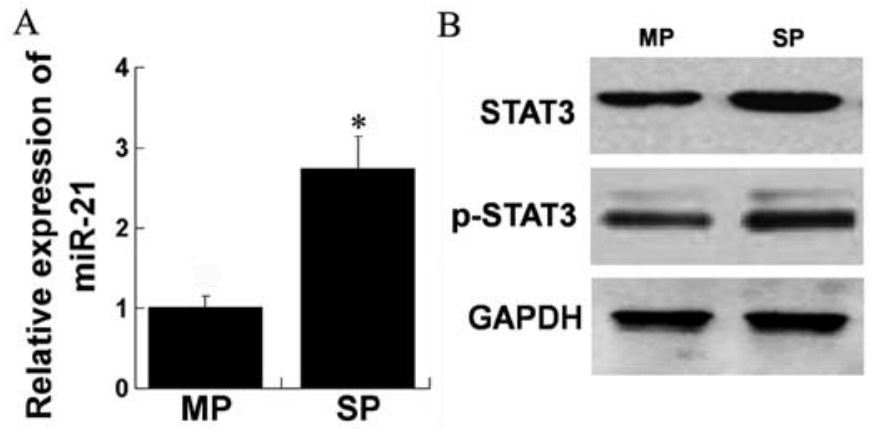

Figure 1. The expression of miR-21, STAT3 and phospho-STAT3. (A) The expression of miR-21 was measured by RT-qPCR. "P<0.05 compared with MP cells. (B) The protein expression levels of STAT3 and phospho-STAT3 were measured by western blotting.
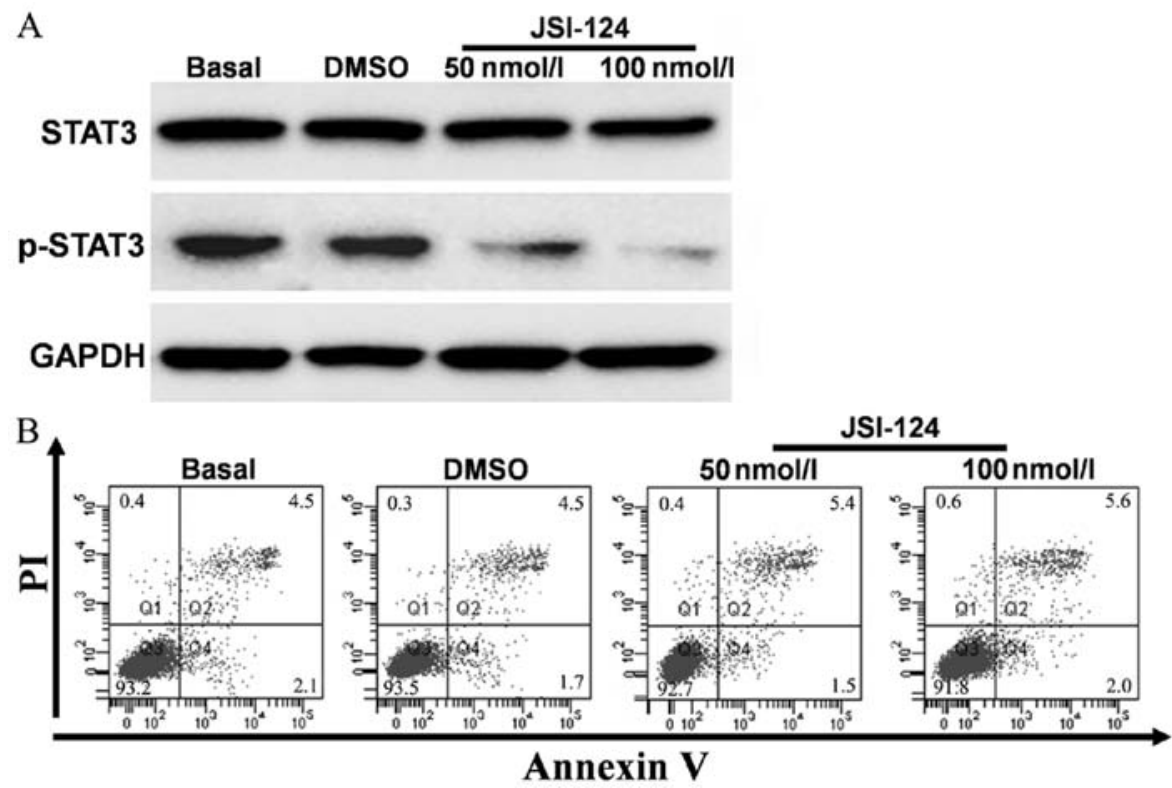

Figure 2. Low concentrations of JSI-124 inhibit phospho-STAT3 without inducing apoptosis. (A) STAT3 and phospho-STAT3 in different concentrations of JSI-124-treated MHCC97H SP cells were detected by western blotting. (B) Apoptosis of different concentrations of cucurbitacin I-treated MHCC97H SP cells were detected by Annexin V/PI analysis.

\section{Results}

MHCC97H cells contain stem-like SP cells. Using flow cytometry, we identified and successfully isolated SP and MP cell populations from MHCC97H cell lines. The SP gate was defined as the region where cells were absent in the presence of verapamil, an agent that blocks the efflux of Hoechst 33342. The SP cells accounted for $2.97 \pm 0.33$ of the total cells in the MHCC97H cell line. The purities of sorted SP and MP cells were $>97 \%$. As previously reported (6), MHCC97H SP cells exhibited stem-like characteristics including tumorigenic potential and chemoresistance migration and invasion abilities, which may lead to relapse and metastasis formation.

STAT3, phospho-STAT3 and miR-21 are overexpressed in HCC SP cells. STAT3 and miR-21 are known to be overexpressed in carcinogenesis and metastasis. We assessed the expression levels of STAT3, phospho-STAT3 and miR-21 in SP cells as compared to MP cells, respectively. RT-qPCR was performed and a higher expression of miR-21 was observed in SP compared to MP cells (Fig. 1A). Western blot results showed that the levels of STAT3 and phospho-STAT3 were higher in SP compared to MP cells (Fig. 1B). These data indicated that STAT3, phospho-STAT3 and miR-21 were overexpressed in SP cells of MHCC97H cell line.

Inhibition of STAT3-regulated metastasis-related capacities of MHCC97H SP cells. Accumulating evidence indicated that STAT3 plays a key role in the maintenance of stem-like cancer cells associated with tumor recurrence, metastasis and chemo-resistance (16). As the effects of STAT3 on HCC SP cells were to be determined, we used JSI-124 to inhibit STAT3 and observed the role of STAT3 on MHCC97H SP cell metastatic capacity.

STAT3 inhibition induced by JSI-124 was evaluated by western blotting. JSI-124 was dissolved in DMSO. The cells were treated with the appropriate volume of PBS or DMSO for the vehicle control. The results showed that phospho-STAT3 protein levels were downregulated significantly when treated with different concentrations of JSI-124 (50 and $100 \mathrm{nM}$ ). The protein levels decreased with the increase of JSI-124 concentrations (Fig. 2A). In order to exclude the possibility 

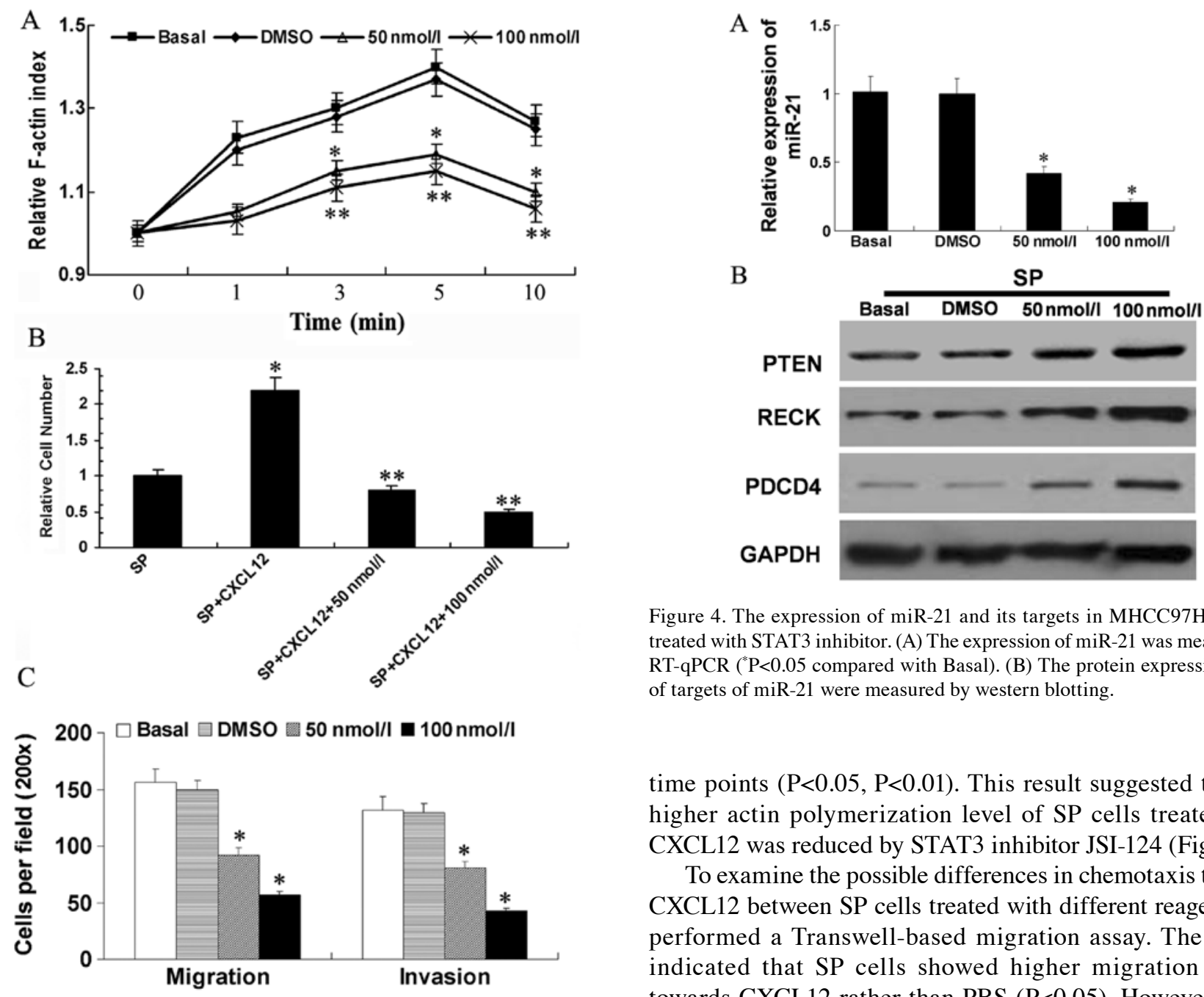

Figure 3. JSI-124 decreased the metastasis-related abilities of MHCC97H SP cells in vitro. (A) F-actin levels of cells treated with different reagents were examined at the indicated time points after CXCL12 stimulation. Phalloidin staining was then analyzed by flow cytometry ( $\left(\mathrm{P}<0.05\right.$ and ${ }^{* * *} \mathrm{P}<0.05$ compared with Basal). (B) The chemotaxis of SP cells treated with or without STAT3 inhibitor towards CXCL12 was examined through a Transwel system $\left({ }^{*} \mathrm{P}<0.05\right.$ compared with $\mathrm{SP},{ }^{* *} \mathrm{P}<0.05$ compared with $\left.\mathrm{SP}+\mathrm{CXCL12}\right)$ (C) The migration and invasion abilities of SP cells treated with or without STAT3 inhibitor were examined through a Transwell system. ("P<0.05 compared with Basal).

that the downregulation of phospho-STAT3 was caused by cell apoptosis, we evaluated JSI-124-treated cells by Annexin V/PI analysis. The results did not indicate significant cell apoptosis in either concentration (Fig. 2B).

Actin polymerization was required by cell polarization. Our previous data indicated that MHCC $97 \mathrm{H}$ SP cells contained higher actin polymerization levels than MP cells when treated with CXCL12. In the present study, we determined whether STAT3 affected actin polymerization of SP cells. As shown in Fig. 3, we treated MHCC97H SP cells with PBS, DMSO and 50 or $100 \mathrm{nM}$ JSI-124, respectively, and then detected the F-actin levels of different cells after 3,5 and 10 min stimulation with CXCL12. Actin polymerization levels of cells treated with PBS or DMSO were similar to each other. Actin polymerization levels of cells treated with 50 or $100 \mathrm{nM}$ JSI-124 were significantly lower than cells treated with PBS at the studied

Figure 4. The expression of miR-21 and its targets in MHCC97H SP cells treated with STAT3 inhibitor. (A) The expression of miR-21 was measured by RT-qPCR (* $\mathrm{P}<0.05$ compared with Basal). (B) The protein expression levels of targets of miR-21 were measured by western blotting.

time points $(\mathrm{P}<0.05, \mathrm{P}<0.01)$. This result suggested that the higher actin polymerization level of SP cells treated with CXCL12 was reduced by STAT3 inhibitor JSI-124 (Fig. 3A).

To examine the possible differences in chemotaxis towards CXCL12 between SP cells treated with different reagents, we performed a Transwell-based migration assay. The results indicated that SP cells showed higher migration ability towards CXCL12 rather than PBS $(\mathrm{P}<0.05)$. However, when treated with 50 or $100 \mathrm{nM}$ JSI-124, the chemotaxis towards CXCL12 decreased significantly $(\mathrm{P}<0.01)$ (Fig. 3B).

We previously reported MHCC97H SP cells exhibited higher abilities of migration and invasion than MP cells and may be important in HCC metastasis. We wondered whether STAT3 affect the metastasis capacities of SP cells. Migration and invasion cell numbers were counted through the Transwell system. The results showed that 50 and $100 \mathrm{nM}$ JSI-124 decreased the SP cell abilities of migration and invasion $(\mathrm{P}<0.05)$ (Fig. 3C).

Inhibition of STAT3 regulated the expression levels of miR-21 and its targets in MHCC $97 \mathrm{H}$ SP cells. It was reported that STAT3 binds to multiple sites in the miR-21 promoter and was required for the induction of transcription in normal or cancer cells $(9,17,18)$. Our data indicated STAT3 phosphorylation affected metastasis-related capacities in vitro of HCC SP cells. We also improved the repression of miR-21-inhibited SP cell migration and invasion in vitro due to upregulation of its target tumor-suppressor genes, i.e., PTEN, RECK or PDCD4. However, whether the effects of phospho-STAT3 on metastasis-related capacities of MHCC97H SP cells were mediated by miR- 21 and its targets remained to be determined. We measured the expression of miR-21 in cells treated with PBS (Basal), DMSO, and 50 or $100 \mathrm{nM}$ JSI-124 through RT-qPCR. It was observed that 50 and $100 \mathrm{nM}$ JSI-124 repressed the expression of miR-21 ( $\mathrm{P}<0.05$, compared with Basal) (Fig. 4A). 
A

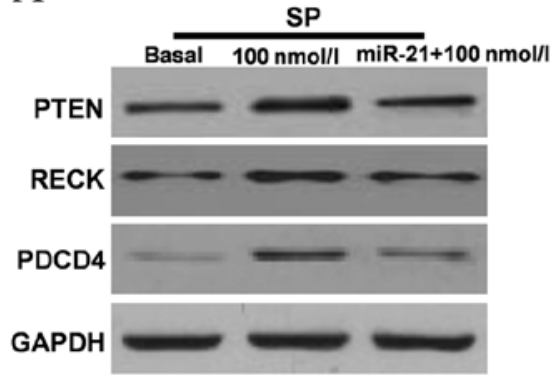

$\mathrm{B}$

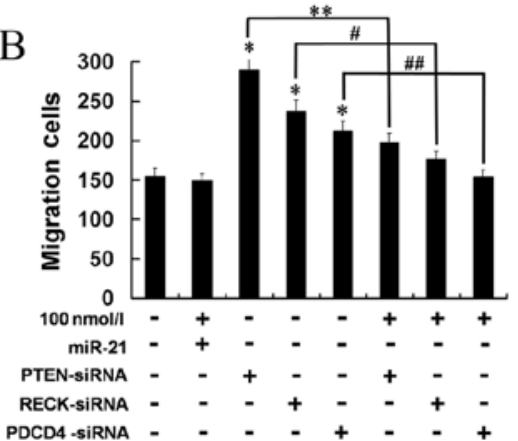

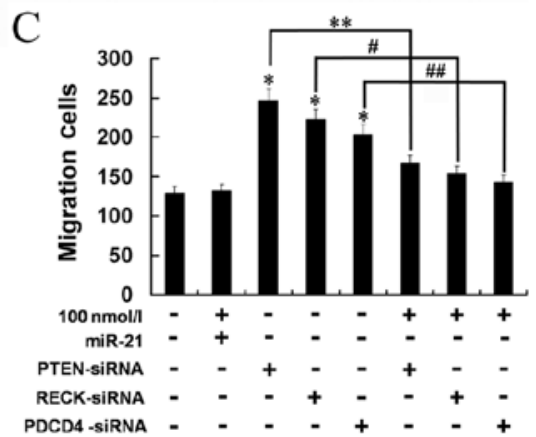

Figure 5. The effects of phospho-STAT3 on migration and invasion of SP cells were partly mediated via miR-21 through its negative regulation of PTEN, RECK or PDCD4 expression. (A) The levels of PTEN, RECK or PDCD4 protein, respectively, in SP cells treated with $100 \mathrm{nM}$ JSI-124 with or without transfection with miR-21 mimics. (B and C) 'Rescue' experiments were developed to investigate the effects of phospho-STAT3, miR-21 and PTEN, RECK or PDCD4 siRNA on SP cell migration and invasion. ("P $<0.05$ compared with non-treated SP cells, ${ }^{* *} \mathrm{P}<0.05$ compared with $100 \mathrm{nM}$ JSI-124 + PTEN-siRNA, ${ }^{*} \mathrm{P}<0.05$ compared with 100 nM JSI-124 + RECK-siRNA, ${ }^{\# \# ~ P<0.05 ~ c o m p a r e d ~ w i t h ~} 100$ nM JSI-124 + PDCD4-siRNA).

Western blot results showed that the levels of PTEN, RECK and PDCD4 were increased with the increasing concentrations of JSI-124 (Fig. 4B).

JSI-124 inhibited phospho-STAT3, while miR-21 and its target gene expression were increased. We determined whether STAT3 was able to regulate PTEN, RECK and PDCD4 via miR-21. MHCC97H SP cells were treated with PBS (Basal), and $100 \mathrm{nM}$ JSI-124 with or without transfection with miR-21 mimics. Transfection with miR-21 mimics led to upregulated miR-21 expression, whereas the increased protein expression of PTEN, RECK and PDCD4 was markedly reduced.

The effects of phospho-STAT3 on migration and invasion of SP cells. We also examined whether inhibition of phospho-STAT3 is involced in the migration and invasion of MHCC97H SP cells via miR-21 and its targets. Western blotting indicated that $100 \mathrm{nM}$ JSI-124 did not induce SP cell migration or invasion when miR-21 expression was upregulated (Fig. 5A). In addition, cell migration and invasion were increased by silencing PTEN, RECK or PDCD4 and these increases were reduced by JSI-124 (all $\mathrm{P}<0.05$ ) (Fig. 5B). These results indicated that the metastatic effect of phospho-STAT3 is partly mediated through miR-21 and its negative regulation of PTEN, RECK and PDCD4 expression.

\section{Discussion}

Accumulating evidence indicated that stem-like cancer cells are the source of malignant phenotypes in many solid tumors. It has also been suggested that stem-like HCC cells play a critical role in initiating and sustaining HCC primary tumors and in facilitating HCC metastasis and recurrence $(24,25)$. SP cells which efflux the DNA-binding dye Hoechst 33342 out of the cell membrane through an ATP-binding cassette (ABC) transporter was first reported in the analysis of hematopoietic stem cells and expanded to various fields. Our previous results (6) showed that SP cells were critical for HCC metastasis because of higher migration and invasion abilities compared to MP cells. Therefore, we determined the potential mechanism involved in HCC SP cell regulation of HCC metastasis.

Abnormal STAT3 signaling, particularly the constitutive activation of STAT3 is important in development and carcinogenesis, since it critically regulates the transcription of multiple key genes involved in cell proliferation, differentiation, apoptosis, angiogenesis, immune response and metastasis $(19,20)$. Activation of STAT3 has been shown to upregulate the expression levels of miRNAs, including miR-21 (21). miR-21 has been shown to be overexpressed in a variety of malignancies, and linked to cell metastasis through its targets PTEN, RECK and PDCD4. PTEN is a phosphoinositide phosphatase acting as a tumor suppressor through Akt and ERK signaling pathways associated with cell survival, proliferation, differentiation, cell migration and invasion. RECK, a membrane-anchored glycoprotein, is a molecular marker for cancer prognosis and controller of cell metastatic capacity (22). Low levels of RECK are often associated with increased invasiveness and poor prognosis $(23,24)$. PDCD4 is a tumor suppressor that inhibits metastasis in human cancer cells (25). It was reported that PDCD4 suppressed the expression and/or activity of the invasion-related proteins such as AKT (26), MAP4K1 (27), and increased the release of metastasis-suppressor proteins such as E-cadherin (28) and TIMP2 (29). We already partly verified that miR-21 regulated MHCC97H SP cell metastasis through its targets PTEN, PDCD4 and RECK. However, whether STAT3 functions as an upstream mediator to regulate the effects of miR-21 and its targets on metastasis in MHCC $97 \mathrm{H}$ SP cells remained to be determined.

To confirm the speculation, we first investigated the expression level of STAT3, phospho-STAT3 and miR-21 of MHCC97H SP and MP cells. A higher expression of STAT3, phospho-STAT3 and miR-21 was observed in SP compared to MP cells. As the critical role of SP cells in HCC malignant phenotypes and the function of STAT3 in HCC survival, proliferation, invasion and angiogenesis $(30,31)$ have been previously proven, we examined the effects of STAT3 on metastasis-related capacities of MHCC97H SP cells.

JSI-124, a chemical compound belonging to the cucurbitacin family, was initally identified as a potent phospho-STAT3 inhibitor in multiple cancer cell lines (18). Inhibition of STAT3 activity was attributed to a disruption in STAT3 DNA-binding activity and gene expression. Thus, we treated MHCC97H SP cells with JSI-124 to observe the effects of STAT3 on SP cell metastatic capacity. Relatively low concentrations of JSI-124 were selected to avoid induction of apparent apoptosis. Western blot and Annexin V/PI analysis show that 50 or $100 \mathrm{nM}$ JSI-124 
did not inhibit STAT3 but phospho-STAT3 effectively, without inducing significant apoptosis. Although SP cells contain a higher metastatic capacity, the present data show that when phospho-STAT3 was inhibited in SP cells, F-actin polymerization, chemotaxis towards CXCL12, migration and invasion cells were weakened, which reduced SP cell metastasis.

miR-21 has been reported to be regulated by an upstream promoter/enhancer containing STAT3 binding sites in malignant disease $(11,12)$. Although we have already confirmed that phospho-STAT3 regulated SP cell chemotaxis, migration and invasion, we determined whether STAT3 regulations were mediated by miR-21 and its targets in MHCC97H SP cells. miR-21 was notably decreased and the protein levels of miR-21 targets PTEN, RECK and PDCD4 were increased when phospho-STAT3 was inhibited. To confirm the relationship between phospho-STAT3 and miR-21, we developed the 'rescue' method. miR-21 expression was upregulated to 'rescue' the inhibitory effect of JSI-124. A marked downregulation was reflected in PTEN, RECK or PDCD4 protein expression. Moreover, Transwell analyses demonstrated that higher migration and invasion abilities gained by silencing the PTEN, RECK or PDCD4 expression could be counteracted by inhibition of phospho-STAT 3 to some extent. These findings further indicated that STAT3 and phospho-STAT3 acted as an upstream regulator of miR-21 in MHCC97H SP cells, as it regulated SP cell metastasis-related capacities through targets of miR-21, PTEN, RECK and PDCD4.

Taken together, the findings provided evidence that STAT3 is crucial in MHCC97H SP cell metastasis via miR-21 and its targets. Consequently, suppression of miR-21 by inhibition of STAT3 may be a novel approach for preventing HCC metastasis.

\section{Acknowledgements}

This study was supported by grants from the National Natural Science Foundation of China (no. 81101619/H1607), the National Natural Science Foundation of Shaanxi Province, China (no. 2012JQ4032), and the National S\&T Major Project for Infectious Diseases of China (no. 2012ZX10002-017).

\section{References}

1. Parkin DM, Bray F, Ferlay J and Pisani P: Global cancer statistics, 2002. CA Cancer J Clin 55: 74-108, 2005.

2. Lau WY and Lai EC: Hepatocellular carcinoma: current management and recent advances. Hepatobiliary Pancreat Dis Int 7: 237-257, 2008.

3. Bjerkvig R, Tysnes BB, Aboody KS, et al: Opinion: the origin of the cancer stem cell: current controversies and new insights. Nat Rev Cancer 5: 899-904, 2005.

4. Visvader JE and Lindeman GJ: Cancer stem cells in solid tumors: accumulating evidence and unresolved questions. Nat Rev Cancer 8: 755-768, 2008.

5. Simeone DM: Pancreatic cancer stem cells: implications for the treatment of pancreatic cancer. Clin Cancer Res 14: 5646-5648, 2008.

6. Zhang N, Li R, Tao KS, et al: Characterization of a stem-like population in hepatocellular carcinoma MHCC97 cells. Oncol Rep 23: 827-831, 2010.

7. Chen J, Wang J, Lin L, et al: Inhibition of STAT3 signaling pathway by nitidine chloride suppressed the angiogenesis and growth of human gastric cancer. Mol Cancer Ther 11: 277-287, 2012.

8. Ho PL, Lay EJ, Jian W, et al: Stat3 activation in urothelial stem cells leads to direct progression to invasive bladder cancer. Cancer Res 72: 3135-3142, 2012.
9. Chen B, Liu J, Chang Q, et al: JNK and STAT3 signaling pathways converge on Akt-mediated phosphorylation of EZH2 in bronchial epithelial cells induced by arsenic. Cell Cycle 12: 112-121, 2013.

10. Cao Q, Li YY, He WF, et al: Interplay between microRNAs and the STAT3 signaling pathway in human cancers. Physiol Genomics 45: 1206-1214, 2013.

11. Iliopoulos D, Jaeger SA, Hirsch HA, et al: STAT3 activation of miR-21 and miR-181b-1 via PTEN and CYLD are part of the epigenetic switch linking inflammation to cancer. Mol Cell 39: 493-506, 2010.

12. Zhou X, Ren Y, Liu A, et al: STAT3 inhibitor WP1066 attenuates miRNA-21 to suppress human oral squamous cell carcinoma growth in vitro and in vivo. Oncol Rep 31: 2173-2180, 2014.

13. Liu C, Yu J, Yu S, et al: MicroRNA-21 acts as an oncomir through multiple targets in human hepatocellular carcinoma. J Hepatol 53: 98-107, 2010.

14. Wang Y, Gao X, Wei F, Zhang X, et al: Diagnostic and prognostic value of circulating miR-21 for cancer: a systematic review and meta-analysis. Gene 533: 389-397, 2014.

15. Zhou L, Yang ZX, Song WJ, et al: MicroRNA-21 regulates the migration and invasion of a stem-like population in hepatocellular carcinoma. Int J Oncol 43: 661-669, 2013.

16. Marotta LL, Almendro V, Marusyk A, et al: The JAK2/STAT3 signaling pathway is required for growth of $\mathrm{CD} 44^{+} \mathrm{CD} 24^{-}$stem cell-like breast cancer cells in human tumors. J Clin Invest 121: 2723-2735, 2011.

17. Bourguignon LY, Earle C, Wong G, Spevak CC and Krueger K: Stem cell marker (Nanog) and Stat-3 signaling promote microRNA-21 expression and chemoresistance in hyaluronan/CD44-activated head and neck squamous cell carcinoma cells. Oncogene 31: 149-160, 2012.

18. Sawant DV, $\mathrm{Wu} \mathrm{H}$, Kaplan $\mathrm{MH}$, et al: The Bcl6 target gene microRNA- 21 promotes Th2 differentiation by a $\mathrm{T}$ cell intrinsic pathway. Mol Immunol 54: 435-442, 2012.

19. Haura EB, Turkson J and Jove R: Mechanisms of disease: insights into the emerging role of signal transducers and activators of transcription in cancer. Nat Clin Pract Oncol 2: 315-324, 2005.

20. Huang S: Regulation of metastases by signal transducer and activator of transcription 3 signaling pathway: clinical implications. Clin Cancer Res 13: 1362-1366, 2007.

21. Löffler D, Brocke-Heidrich K, Pfeifer G, et al: Interleukin-6 dependent survival of multiple myeloma cells involves the Stat3-mediated induction of microRNA-21 through a highly conserved enhancer. Blood 110: 1330-1333, 2007.

22. Takahashi C, Sheng Z, Horan TP, et al: Regulation of matrix metalloproteinase- 9 and inhibition of tumor invasion by the membrane-anchored glycoprotein RECK. Proc Natl Acad Sci USA 95: 13221-13226, 1998.

23. Kotzsch M, Farthmann J, Meye A, et al: Prognostic relevance of uPAR-del4/5 and TIMP-3 mRNA expression levels in breast cancer. Eur J Cancer 41: 2760-2768, 2005.

24. Takenaka K, Ishikawa S, Kawano Y, et al: Expression of a novel matrix metalloproteinase regulator, RECK and its clinical significance in resected non-small cell lung cancer. Eur J Cancer 40: 1617-1623, 2004.

25. Allgayer H: Pdcd4, a colon cancer prognostic that is regulated by a microRNA. Crit Rev Oncol Hematol 73: 185-191, 2010.

26. Lankat-Buttgereit B and Goke R: The tumour suppressor Pdcd4: recent advances in the elucidation of function and regulation. Biol Cell 101: 309-317, 2009.

27. Yang HS, Matthews CP, Clair T, et al: Tumorigenesis suppressor Pdcd4 down-regulates mitogen-activated protein kinase kinase kinase kinase 1 expression to suppress colon carcinoma cell invasion. Mol Cell Biol 26: 1297-1306, 2006.

28. Wang Q, Sun Z and Yang HS: Downregulation of tumor suppressor Pdcd4 promotes invasion and activates both beta-catenin/Tcf and AP-1-dependent transcription in colon carcinoma cells. Oncogene 27: 1527-1535, 2008.

29. Nieves-Alicea R, Colburn NH, Simeone AM, et al: Programmed cell death 4 inhibits breast cancer cell invasion by increasing tissue inhibitor of metalloproteinase- 2 expression. Breast Cancer Res Treat 114: 203-209, 2009.

30. Rajendran P, Li F, Shanmugam MK, et al: Celastrol suppresses growth and induces apoptosis of human hepatocellular carcinoma through the modulation of STAT3/JAK2 signaling cascade in vitro and in vivo. Cancer Prev Res 5: 631-643, 2012.

31. Meydan N, Grunberger T, Dadi H, et al: Inhibition of acute lymphoblastic leukaemia by a Jak-2 inhibitor. Nature 13: 645-648, 1996. 